University of South Carolina

Scholar Commons

\title{
Ni-Composite Microencapsulated Graphite as the Negative Electrode in Lithium-Ion Batteries II: Electrochemical Impedance and Self-Discharge Studies
}

\author{
P. Yu \\ University of South Carolina - Columbia \\ James A. Ritter \\ University of South Carolina - Columbia \\ Ralph E. White \\ University of South Carolina - Columbia, white@cec.sc.edu \\ Branko N. Popov \\ University of South Carolina - Columbia, popov@engr.sc.edu
}

Follow this and additional works at: https://scholarcommons.sc.edu/eche_facpub

Part of the Chemical Engineering Commons

\section{Publication Info}

Journal of the Electrochemical Society, 2000, pages 2081-2085.

(c) The Electrochemical Society, Inc. 2000. All rights reserved. Except as provided under U.S. copyright law, this work may not be reproduced, resold, distributed, or modified without the express permission of The Electrochemical Society (ECS). The archival version of this work was published in the Journal of the Electrochemical Society.

http://www.electrochem.org/

Publisher's link: http://dx.doi.org/10.1149/1.1393489

DOI: $10.1149 / 1.1393489$

This Article is brought to you by the Chemical Engineering, Department of at Scholar Commons. It has been accepted for inclusion in Faculty Publications by an authorized administrator of Scholar Commons. For more information, please contact digres@mailbox.sc.edu. 


\title{
Ni-Composite Microencapsulated Graphite as the Negative Electrode in Lithium-Ion Batteries
}

\section{Electrochemical Impedance and Self-Discharge Studies}

\author{
P. Yu,* J. A. Ritter,** R. E. White, ${ }^{* * * *}$ and B. N. Popov**,z \\ Center for Electrochemical Engineering, Department of Chemical Engineering, University of South Carolina, Columbia, \\ South Carolina 29208, USA
}

\begin{abstract}
Electrochemical impedance and self-discharge studies were carried out to investigate lithium intercalation into bare and Ni-coated KS10 graphite. Values of the charge-transfer resistances, exchange current densities, surface film resistances, and lithium-ion diffusion coefficients as functions of the state of charge (SOC) all favored the $10 \mathrm{wt} \% \mathrm{Ni}$ composite KS10 graphite over bare KS10 graphite when these materials were used as the negative electrode in a Li-ion cell with mixed organic electrolyte. The charge-transfer resistances were always lower and gave rise to between 26 and $27 \%$ larger exchange current densities, which increased from 137 to $614 \mathrm{~mA} / \mathrm{g}$ as the SOC increased. The surface film resistances for Ni composite KS10 were between 0.02 and $0.05 \Omega \mathrm{g}$, slightly smaller than those of 0.03 to $0.08 \Omega \mathrm{g}$ for bare KS10, and both surface film resistances decreased with increasing SOC. The lithium-ion diffusion coefficients were always slightly larger, ranging between $1.09 \times 10^{-9}$ and $6.7 \times 10^{-9} \mathrm{~cm}^{2} / \mathrm{s}$. Results from the self-discharge study also favored the $10 \mathrm{wt} \% \mathrm{Ni}$ composite KS10, which exhibited less capacity loss over a 10 day period compared to bare KS10.
\end{abstract}

(c) 2000 The Electrochemical Society. S0013-4651(99)09-057-6. All rights reserved.

Manuscript submitted September 16, 1999; revised manuscript received February 1, 2000.

A considerable amount of research has been carried out to develop carbonaceous materials for the lithium-ion battery that have a high reversible capacity and to investigate the structural changes that take place during lithium intercalation into these carbonaceous materials. However, relatively few studies have focused on kinetic aspects, such as determination of the electrochemical exchange current density and the lithium-ion diffusion coefficient, despite their importance in battery charge/discharge characteristics. ${ }^{5-7}$ The exchange current density characterizes the electrocatalytic activity of the electrode, which controls the kinetics of the charge-transfer reaction occurring at the electrode/electrolyte interface. In contrast, the lithium-ion diffusion coefficient determines the charge/discharge performance, particularly at high charge/discharge rates, because the diffusion of lithium-ions into and out of the carbon structure are the rate-determining steps during charge and discharge, in most cases. ${ }^{8}$ Clearly, the determination of these kinetic parameters is essential for predicting and optimizing the performance of new carbonaceous materials being developed for use in the lithium-ion battery.

Part I of this work ${ }^{9}$ reported on the development of a novel electroless deposition procedure for making Ni composite graphite materials for use as the anode (negative electrode) in the lithium-ion battery. These materials significantly suppressed solvated lithium intercalation into the graphite in propylene carbonate (PC)-based electrolyte, decreased the irreversible capacity in the first cycle, and exhibited 30 40 mAh/g higher discharge capacities (delithiation) compared to bare graphite at the same rate. However, as mentioned above, a superior anode material must also exhibit good kinetics. Therefore, the objective of Part II of this work is to report on the kinetic effects due to the presence of a $\mathrm{Ni}$ composite coating on graphite. To reveal these effects, electrochemical charge-transfer resistances, exchange current densities, surface film resistances, and lithium-ion diffusion coefficients are determined for the $10 \mathrm{wt} \% \mathrm{Ni}$ composite KS10 graphite developed in Part I and also for the bare KS10 graphite precusor. The self-discharge behavior of these two materials is also investigated to determine the role of the Ni composite coating.

\section{Experimental}

The microencapsulation of the graphite $\mathrm{KS} 10$ (10 $\mu \mathrm{m}$, Timcal) with Ni composite was carried out between 85 and $90^{\circ} \mathrm{C}$ in a plating

\footnotetext{
* Electrochemical Society Student Member.

** Electrochemical Society Active Member.

*** Electrochemical Society Fellow.

${ }^{\mathrm{z}}$ E-mail: popov@engr.sc.edu
}

bath containing sodium hypophosphite as a reducing agent. The nickel composite coating consisted of nanosized particles distributed over the surface of the graphite particle. ${ }^{9}$ The thickness of the Ni composite coating was calculated to be approximately $3.4 \mathrm{~nm} .{ }^{9}$ Therefore, there was no marked change in the graphite structure or the particle size of KS10 $(10 \mu \mathrm{m})$ after microencapsulation. However, the Brunauer-Emmett-Teller (BET) surface area of the $10 \mathrm{wt} \%$ Ni composite KS10 decreased slightly from $14.5 \mathrm{~m}^{2} / \mathrm{g}$ (estimated for bare $\mathrm{KS} 10$ ) to $12.3 \mathrm{~m}^{2} / \mathrm{g}$. In this work, $10 \mathrm{wt} \% \mathrm{Ni}$ composite KS10 and bare KS10 graphites were electrochemically characterized to obtain various kinetic parameters.

The electrochemical characterizations of these materials were carried out using a Swagelok three-electrode T-cell. A typical graphite negative electrode was prepared by mixing graphite powder with $6 \mathrm{wt}$ $\%$ poly(vinylidene fluoride) (PVDF, Aldrich) powder and 1-methyl2-pyrrolidinone solvent. The resulting slurry was spread onto a stainless steel current collector and dried under vacuum at $150^{\circ} \mathrm{C}$ for $12 \mathrm{~h}$. The pellet was approximately $95 \mu \mathrm{m}$ in thickness, $1.26 \mathrm{~cm}$ in diameter, and contained about $9 \mathrm{mg}$ of material. Because Markovsky et al. ${ }^{10}$ showed that both the kinetics and impedance of the composite electrode were affected by the amount of binder, particle size and orientation, and electrode thickness, the kinetic behavior of bare and Ni composite KS10 was studied using electrode pellets prepared with approximately the same thickness, weight, and amount of binder. Taking into account that the properties of KS10 graphite particles are similar to those of KS6 $(6 \mu \mathrm{m})$, which was found to be highly oriented, ${ }^{10}$ the above preparation procedure yields a horizontal orientation of the particles with their basal planes parallel to the current collector. This horizontal orientation of the KS10 particles was observed in scanning electron microscopy (SEM) images. ${ }^{9}$

The counter and reference electrodes were made from lithium foil (99.9\%, Aldrich), and a sheet of Whatman glass fiber membrane (Baxter Diagnostics Co.) was used as a separator. The electrolyte consisted of $1 \mathrm{M} \mathrm{LiPF}_{6}$ in a 1:1:3 mixture of propylene carbonate/ ethylene carbonate/dimethyl carbonate (PC, EC, DMC) with less than $15 \mathrm{ppm} \mathrm{H}_{2} \mathrm{O}$ and $80 \mathrm{ppm} \mathrm{HF}$ from (EM Inc.).

Electrochemical impedance spectroscopy (EIS) was carried out at different states of charge at room temperature. Before the EIS experiments were performed, each T-cell was initially charged and discharged between 0.005 and $1.0 \mathrm{~V} \mathrm{vs.} \mathrm{Li}^{+} / \mathrm{Li}$ for three cycles using a constant current density of $0.5 \mathrm{~mA} / \mathrm{cm}^{2}$ to stabilize the system and to avoid the influence of any irreversible phenomena occurring during the first cycle. The electrochemical impedance data were gener- 
ated in the frequency range of $100 \mathrm{kHz}$ to $0.005 \mathrm{~Hz}$ with a low ac voltage amplitude of $5 \mathrm{mV}$ to minimize perturbation of the system. Self-discharge behavior was examined using the following procedure. Each T-cell was first cycled three times, then charged at $\mathrm{C} / 8$ rate to $0.005 \mathrm{Vvs}$. $\mathrm{Li}^{+} / \mathrm{Li}$, held under open-circuit conditions for a period of time, and finally discharged at the same rate $(\mathrm{C} / 8)$ to $2 \mathrm{~V}$ $v s . \mathrm{Li}^{+} / \mathrm{Li}$. The discharge capacity after storage was measured, and the percent retained was determined by the ratio of this discharge capacity to the initial discharge capacity. All experiments were carried out at $25^{\circ} \mathrm{C}$ using an EG\&G PAR potentiostat/galvanostat model 273A and SI 1255 frequency response analyzer driven by the CorrWare software system from Scribner Associates, Inc. Also, the electrical conductivities of the materials were measured by pressing about $0.7 \mathrm{~g}$ of powder into a pellet $0.8 \mathrm{~cm}$ in diameter and $6 \mathrm{~mm}$ thick at $65,000 \mathrm{psi}$ and then measuring the electric resistance of the pellet using a digital multimeter.

\section{Results and Discussion}

Figures $1 \mathrm{a}$ and $\mathrm{b}$ display the Nyquist plots obtained from the EIS experiments for bare KS10 and $10 \mathrm{wt} \% \mathrm{Ni}$ composite KS10 at various states of charge (SOC), respectively. All of the spectra for both materials exhibit at least one semicircle and most exhibit two semicircles, which are associated with nondiffusive impedance. The impedance is explained in Fig. 2 in terms of a generalized equivalent
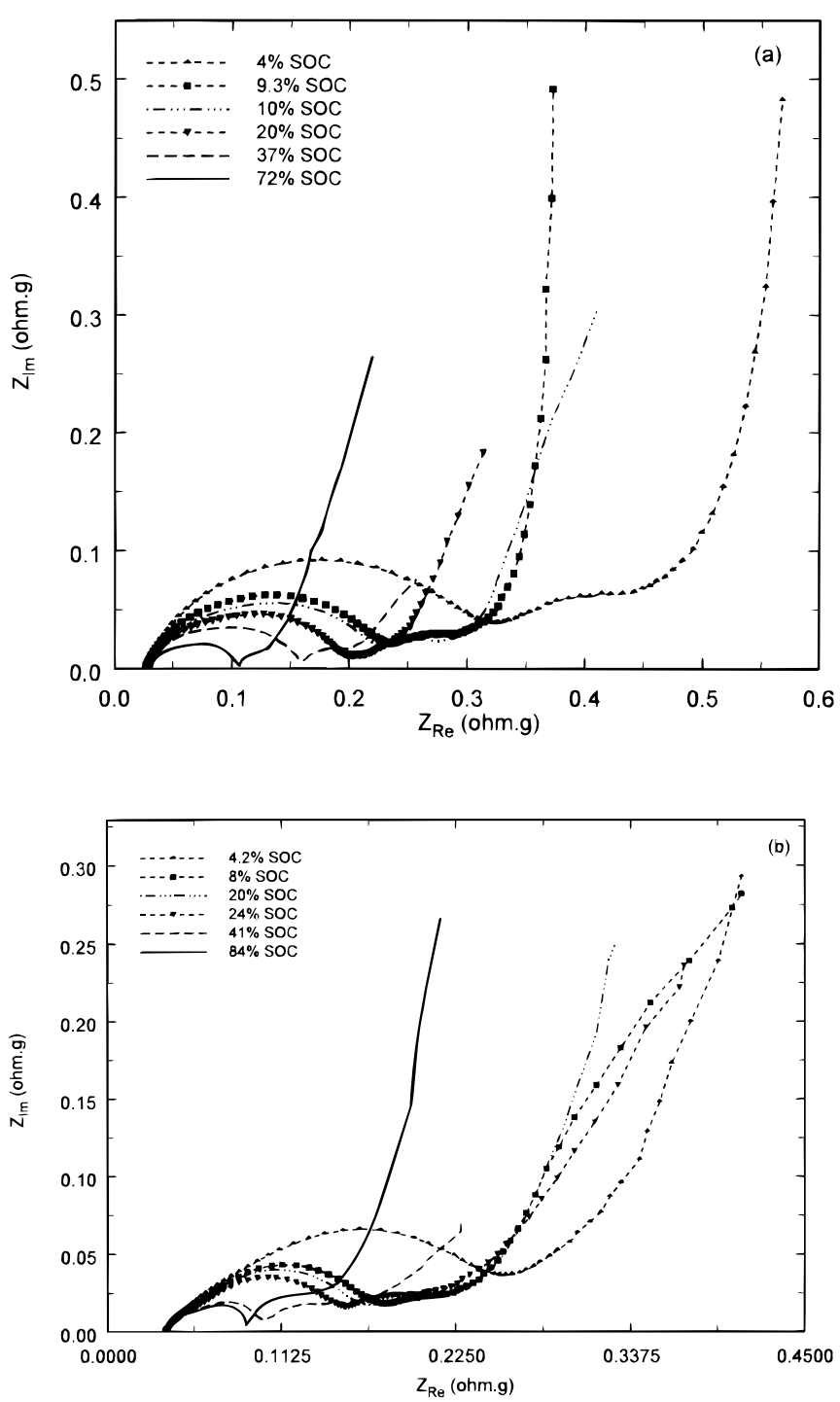

Figure 1. Nyquist plots for (a) bare KS10 and (b) 10 wt \% Ni composite $\mathrm{KS} 10$ at various SOC.

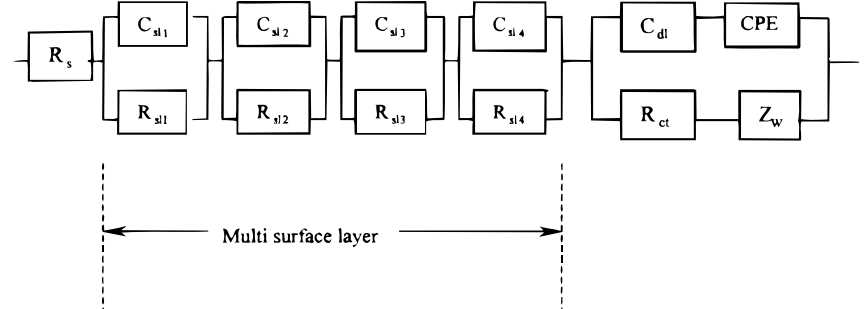

Figure 2. Equivalent circuit based on the impedance spectra in Fig. 1.

circuit. The first semicircle occurring in the high-frequency region is a combination of $\mathrm{R} / / \mathrm{C}$ circuits in series and represents the migration of Li ions through the passivation films. ${ }^{10}$ The surface species are precipitated in a gradual reduction process at the active reductive electrode surface. Thus, the passivation films have a multilayer structure due to the variation of composition of the surface passivating species with distance from the active electrode material. ${ }^{11}$ The $\mathrm{R} / / \mathrm{C}$ circuits in series are used to characterize this phenomenon with the total resistance $R_{\mathrm{sl}}$ and total capacitance $C_{\mathrm{sl}} \cdot{ }^{10,12}$ The second semicircle in the medium-frequency region is attributed to an interfacial charge transfer of $\mathrm{Li}^{+} / \mathrm{Li}$ with a resistance $R_{\mathrm{ct}}$ and doublelayer capacitance $C_{\mathrm{dl}}$. The capacitive component, denoted as a constant-phase element (CPE), is introduced into the circuit to describe the depressed nature of the semicircle. In addition, $R_{\mathrm{S}}$ accounts for the ionic resistance of the electrolyte in the generalized electric circuit. It is interesting that for both materials the sizes of the semicircles decrease with an increase in the SOC. This behavior is indicative of a decrease in the nondiffusive impedance as more lithium intercalates into the graphite.

The low-frequency spectra beyond the semicircles are attributed to $\mathrm{Li}^{+}$diffusion impedance. The $45^{\circ}$ slope in the initial parts of these spectra corresponds to semi-infinite diffusion, which is denoted by the Warburg impedance $Z_{\mathrm{w}}$ and included in the equivalent circuit in series with the charge transfer. Spectra at the very low frequencies, with slopes steeper than $45^{\circ}$ and in some cases even approaching $90^{\circ}$ (vertical spectra), are associated with finite diffusion. The region between the finite diffusion and semi-infinite diffusion regions, i.e., the transition region, has been used very effectively to obtain the diffusion coefficient, ${ }^{11,12}$ as shown below.

The equivalent circuit depicted in Fig. 2 was regressed to the impedance data using Solatron impedance software, Zview ${ }^{\mathrm{TM}}$ (Scribner Associates, Inc.), to obtain the different resistances and capacitances as a function of the SOC. The resulting surface film resistances $R_{\mathrm{sl}}$ at various SOCs are presented in Fig. 3 for both bare KS10 and for 10 wt $\%$ Ni composite KS10. For both materials, $R_{\mathrm{sl}}$ decreases slightly with increasing SOC. The values of $R_{\mathrm{sl}}$ for bare KS10 range from 0.08 to $0.3 \Omega \mathrm{g}$, or from 11.2 to $42 \Omega \mathrm{cm}^{2}$. The evaluation of the resis-

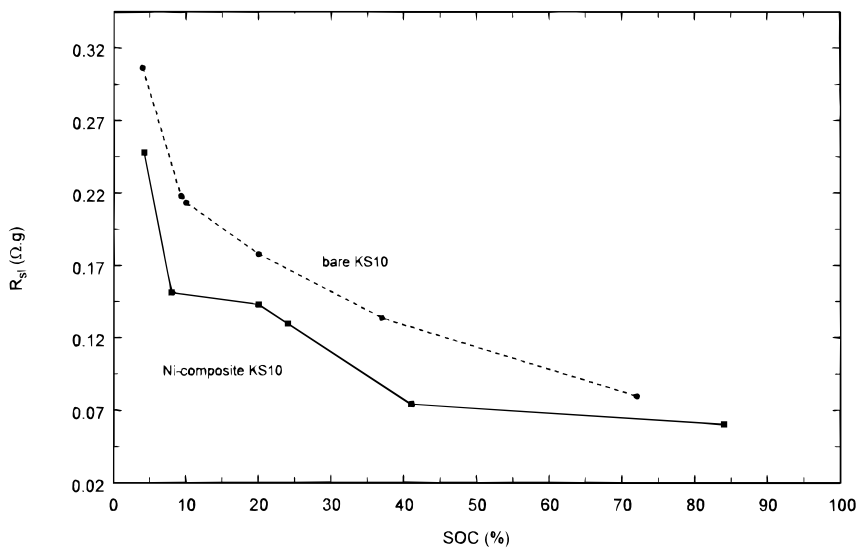

Figure 3. Surface film resistance extracted from the data in Fig. 1 as a function of SOC for bare KS10 and $10 \mathrm{wt} \% \mathrm{Ni}$ composite KS10. 
tance was based on the geometric surface area of $1.26 \mathrm{~cm}^{2}$ or on the pellet weight of $9 \mathrm{mg}$. The observed surface film resistances are comparable to those reported in the literature for lithium metal ( 7 to $49 \Omega$ $\left.\mathrm{cm}^{2}\right) .{ }^{13}$ The $R_{\mathrm{sl}}$ values evaluated for Ni composite KS10 varied between 0.02 and $0.05 \Omega \mathrm{cm}^{2}$. These values are consistently smaller than those observed for bare KS10. These results indicate that the presence of Ni composite particles on KS10 graphite reduces the surface film resistance of bare KS10. The observed decrease in the surface film resistance may arise from the enhanced ionic conductivity of the surface film around grain boundaries due to the Ni composite coating because nickel metal has a larger conductivity $\left[1.44 \times 10^{5}(\Omega\right.$ $\left.\left.\mathrm{cm}^{-1}\right)\right]$ than graphite $\left[7.34 \times 10^{4}(\Omega \mathrm{cm})^{-1}\right] .{ }^{14}$

In addition, the Ni composite coating on KS10 significantly suppresses solvated lithium intercalation and reduction. ${ }^{9}$ Thus, a smaller amount of electrolyte is reduced on the Ni composite KS10 resulting in a thinner surface film when compared to the bare graphite; this leads to a lower surface film resistance.

The dependence of the charge-transfer resistance $R_{\mathrm{ct}}$ on SOC is plotted in Fig. 4 for both bare KS10 and $10 \mathrm{wt} \% \mathrm{Ni}$ composite KS10. For both materials, $R_{\mathrm{ct}}$ decreases slightly with increasing $\mathrm{SOC}$, i.e., as more lithium-ions intercalate into the graphite. However, $R_{\mathrm{ct}}$ for bare KS10 is consistently higher. For example, $R_{\mathrm{ct}}$ for bare KS10 ranged between 0.065 and $0.246 \Omega \mathrm{g}$, or between 9.4 and $35.7 \mathrm{k} \Omega \mathrm{cm}^{2}$ based on a specific surface area (BET) of $14.5 \mathrm{~m}^{2} / \mathrm{g}$ for bare KS10. ${ }^{9}$ These values are larger than those reported in the literature at relatively high SOC for a natural graphite. ${ }^{7} R_{\mathrm{ct}}$ values for the 10 wt $\%$ Ni composite KS10 were between 0.056 and $0.173 \Omega \mathrm{g}$, or between 6.8 and $21.3 \mathrm{k} \Omega \mathrm{cm}^{2}$ based on a BET surface area of $12.3 \mathrm{~m}^{2} / \mathrm{g}$ for $10 \mathrm{wt} \% \mathrm{Ni}$ composite KS $10 .^{9}$

These results indicate that the presence of a $10 \mathrm{wt} \% \mathrm{Ni}$ composite coating on KS10 graphite reduces the charge-transfer resistance compared to bare KS10 at all SOCs. The charge-transfer resistances in Fig. 4 are also used to determine the exchange current density $i_{0}$, which characterizes the electrocatalytic activity of the negative electrode.

According to a linearized version of the Butler-Volmer equation at low overpotentials, ${ }^{15} i_{\mathrm{o}}$ is determined from

$$
R_{\mathrm{ct}}=\frac{R T}{F i_{0}}
$$

where $T$ is the absolute temperature $(298 \mathrm{~K}), R$ is the universal gas constant, and $F$ is Faraday's constant. The corresponding exchange current densities for bare KS10 and $10 \mathrm{wt} \% \mathrm{Ni}$ composite KS10 are plotted as a function of the SOC in Fig. 5. Because of the inverse relation between $R_{\mathrm{ct}}$ and $i_{\mathrm{o}}$, the decreasing charge-transfer resistance noted in Fig. 5 produces an increasing exchange current density with increasing SOC; similarly, because $R_{\mathrm{ct}}$ is larger for bare $\mathrm{KS} 10, i_{\mathrm{o}}$ is

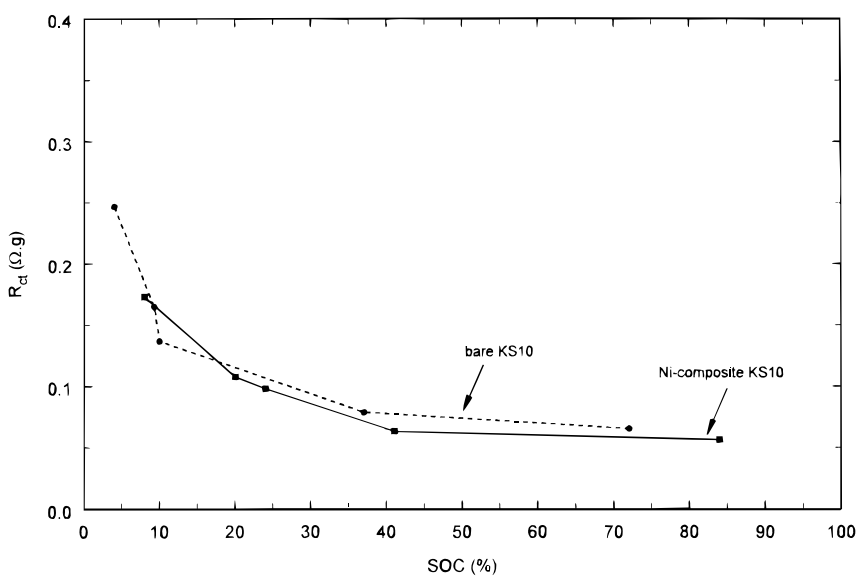

Figure 4. Charge-transfer resistance extracted from the data in Fig. 1 as a function of SOC for bare KS10 and 10 wt \% Ni composite KS10. smaller. For example, $i_{0}$ ranges between 148 and $454 \mathrm{~mA} / \mathrm{g}$ for $10 \mathrm{wt}$ $\% \mathrm{Ni}$ composite KS10 and only between 104 and $391 \mathrm{~mA} / \mathrm{g}$ for bare KS10.

These consistently higher values of $i_{\mathrm{o}}$ for the $10 \mathrm{wt} \% \mathrm{Ni}$ composite KS10 are consistent with $R_{\mathrm{ct}}$ depending strongly on the composition of the surface. ${ }^{16}$ These results also indicate that the electrocatalytic activity of the KS10 graphite surface coated with Ni composite is more favorable toward the $\mathrm{Li}^{+} / \mathrm{Li}$ charge-transfer reaction than the bare KS10 graphite surface. The charge-transfer reaction rate is also affected by and decreases with an increase in the electrical resistance of the electrode material, which is about $14 \%$ higher for bare $\mathrm{KS} 10$ (3.3 $\Omega$ ) compared to $10 \mathrm{wt} \% \mathrm{Ni}$ composite $\mathrm{KS} 10$ $(2.9 \Omega)$. This result illustrates that the electrical conductivity and thus the charge-transfer reaction rate both increase by coating KS10 graphite with $10 \mathrm{wt} \% \mathrm{Ni}$ composite. This improvement is most likely due to the higher conductivity of Ni metal compared to graphite, as shown above. Due to the higher charge-transfer reaction rate associated with the higher conductivity, a higher reversible capacity is also manifest in the $10 \mathrm{wt} \% \mathrm{Ni}$ composite KS10 during long-time cycling, as illustrated in Part I of this series. ${ }^{9}$

The lithium-ion diffusion in graphite is a very important transport step among a series of processes that take place sequentially during charging. ${ }^{17}$ For example, lithium-ions (i) must diffuse through the electrolyte, (ii) migrate through the SEI film, (iii) electrochemically react via charge transfer on the surface, and (iv) diffuse through and intercalate between the graphene layers within the graphite structure. Therefore, it is critical to determine the lithium-ion diffusion coefficient with some degree of accuracy. Different techniques have been explored for obtaining the lithium-ion diffusion coefficient; the advantages and disadvantages of each have been presented in the previous paper. ${ }^{11}$ Based on some previous work by the authors, ${ }^{11,12}$ the slopes of the impedance spectra in the transition regions of the Nyquist plots (Fig. 1a and b) were analyzed with the assistance of the following equations

$$
\begin{gathered}
Z(\omega)=\frac{\partial \eta_{\mathrm{R}}}{\partial I}+\frac{(1-i) \sigma}{\sqrt{\omega}\{\operatorname{coth}[(1+j) \psi]-(1-j) \psi\}^{1 / 2}} \\
\frac{d\left(Z_{\mathrm{Im}}\right)}{d\left(Z_{\mathrm{Re}}\right)}=\frac{\left(S_{3} S_{5}+S_{4} S_{7}-S_{1} S_{6}+S_{2} S_{8}\right) T_{4}-2 T_{3}\left(S_{4} S_{3}+S_{2} S_{1}\right)}{\left(S_{3} S_{6}+S_{4} S_{8}-S_{1} S_{5}+S_{2} S_{7}\right) T_{4}-2 T_{5}\left(S_{4} S_{3}+S_{2} S_{1}\right)} \\
T_{3}=\left(S_{4} S_{5}-S_{2} S_{6}\right) ; \quad T_{4}=\left(S_{4}^{2}+S_{2}^{2}\right) ; \quad T_{5}=\left(S_{4} S_{6}+S_{2} S_{5}\right) \\
S_{1}=S_{5} S_{6} ; \quad S_{2}=2 \psi-S_{5} ; \quad S_{3}=2 \operatorname{coth}(\psi) \cot (\psi)\left(1-\psi S_{6}\right)
\end{gathered}
$$

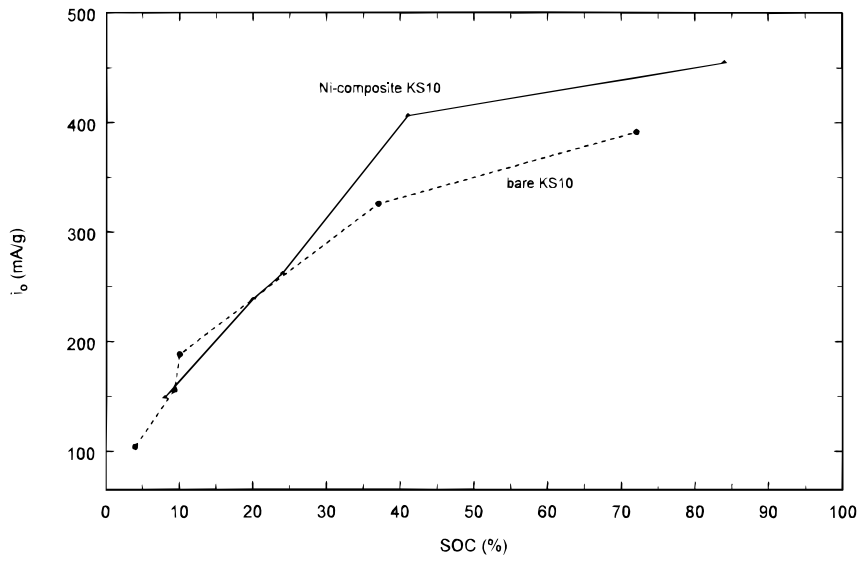

Figure 5. Exchange current density calculated as a function of SOC for bare KS10 and 10 wt \% Ni composite KS10. 


$$
\begin{gathered}
S_{4}=2 \psi \operatorname{coth}(\psi) \cot (\psi)-S_{6} ; \quad S_{5}=\operatorname{coth}(\psi)-\cot (\psi) \\
S_{6}=\operatorname{coth}(\psi)+\cot (\psi) ; S_{7}=2-S_{1} ; \quad S_{8}=\cot (\psi)^{2}+\operatorname{coth}(\psi)^{2}[7] \\
\psi=\sqrt{\frac{\omega R_{\mathrm{p}}^{2}}{2 D}}
\end{gathered}
$$

where $Z(\omega)$ is the complex electrochemical impedance that includes $\partial \eta_{\mathrm{R}} / \partial I$ as the nondiffusion impedance (i.e., the charge-transfer resistance and the surface film resistance) and $(1-j) \sigma / \sqrt{\omega}[\operatorname{coth}(1+j) \psi-$ $(1-j) \psi]^{1 / 2}$ as the diffusion impedance. $\sigma$ is the modified Warburg prefactor, $j$ is $\sqrt{-1}, D$ is the lithium-ion diffusion coefficient in graphite, $\omega$ is the angular frequency, and $R_{\mathrm{p}}$ is the particle radius $(5 \mu \mathrm{m}$ for KS10). The resulting diffusion coefficients for bare KS10 and $10 \mathrm{wt}$ $\% \mathrm{Ni}$ composite KS10 are presented in Fig. 6 as a function of SOC.

The lithium-ion diffusion coefficients obtained for both bare KS10 and 10 wt \% Ni composite KS10 are comparable to each other and vary similarly with SOC. In most cases, however, the lithiumion diffusion coefficient is slightly higher for the $10 \mathrm{wt} \% \mathrm{Ni} \mathrm{com}-$ posite KS10 with values ranging from $1.3 \times 10^{-9}$ to $7.1 \times$ $10^{-9} \mathrm{~cm}^{2} / \mathrm{s}$, the corresponding values for bare $\mathrm{KS} 10$ range from $1.1 \times 10^{-9}$ to $6.7 \times 10^{-9} \mathrm{~cm}^{2} / \mathrm{s}$. These values are comparable to those reported by Guyomard and Tarascon ${ }^{18}$ for petroleum coke $\left(1.0 \times 10^{-9}\right.$ to $\left.1.8 \times 10^{-8} \mathrm{~cm}^{2} / \mathrm{s}\right)$ using the potential intermittent titration technique (PITT). These results show very clearly that the $10 \mathrm{wt} \% \mathrm{Ni}$ composite coating on KS10 graphite does not hinder the diffusion of lithium-ions into the graphite structure, and in most cases it has a beneficial effect.

It is also interesting that for both materials the lithium-ion diffusion coefficients exhibit minimum values at about $20 \%$ SOC. In other words, at low SOCs the lithium-ion diffusion coefficient decreases with increasing SOC, which is in agreement with that reported for other carbonaceous materials. ${ }^{18-20}$ However, at SOCs above $20 \%$, the lithium-ion diffusion coefficient begins to increase with SOC. An increase in the lithium-ion diffusion coefficient at higher SOCs has been reported by Funabiki et al. ${ }^{7}$ for a natural graphite and by Julien ${ }^{21}$ for fluorographite $\mathrm{CF}_{1.13}$. This phenomenon can be attributed to the formation of different staged compounds, i.e., different graphite intercalation compounds (GICs), as the lithium-ion insertion process continues.

The dependence of the lithium-ion diffusion coefficient on the staged structure of graphite can be ascribed ${ }^{7}$ to differences in the inplane and out-of-plane Li-GICs, because stage 1 and stage 2 phases (high SOC) are known to have an in-plane superstructure, ${ }^{22}$ whereas stage 2 , stage 3 , and stage 4 phases (medium SOC) do not. ${ }^{7}$ As expected, the transport of lithium ions in an in-plane structure is much more rapid than in an out-of-plane structure. Moreover, it has

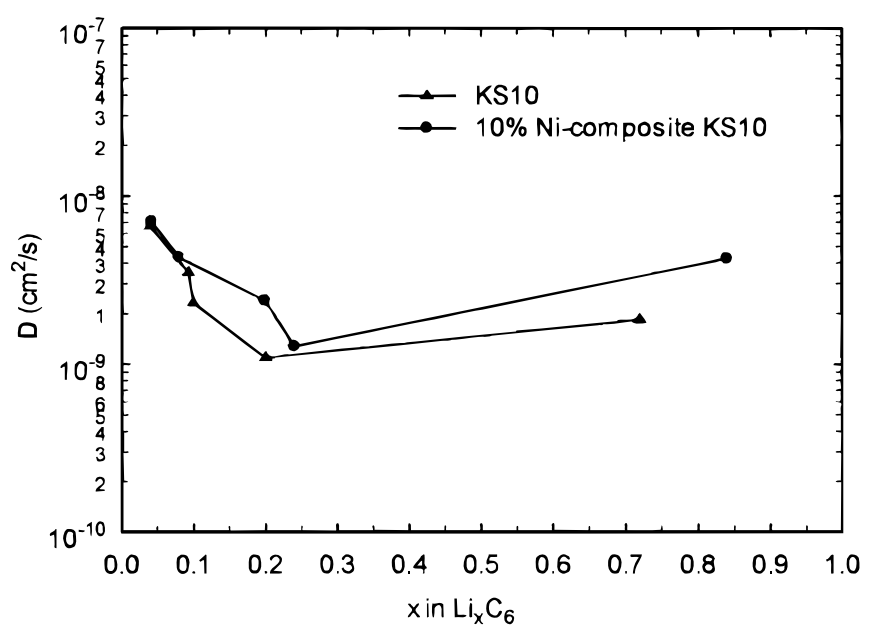

Figure 6. Lithium-ion diffusion coefficients extracted from the data in Fig. 1 as a function of SOC for bare KS10 and $10 \mathrm{wt} \% \mathrm{Ni}$ composite KS10. also been shown ${ }^{21}$ that local strains are greatly reduced by the coinsertion of fluorine and lithium-ions into the host graphite structure, which is beneficial to the enhanced in-plane diffusion coefficients. In addition, the graphene layer undergoes a large expansion in the $c$ axis direction during the formation of the stage 1 compounds (high SOC). In contrast, much less expansion occurs during the formation of stage 3 compounds (medium SOC). ${ }^{8}$ This large expansion of the graphene layer, corresponding to an enlarged layer spacing, most likely gives rise to the increased lithium-ion diffusion coefficients at high SOCs as the diffusion of lithium-ions takes place in the direction parallel to the graphene layer. These three features described above most likely contribute to the increased lithium ion diffusion coefficients observed for both bare KS10 and $10 \mathrm{wt} \%$ Ni composite KS10 at higher SOCs.

In addition, the abrupt variation of the diffusion coefficient exhibited at about 20\% SOC for both bare KS10 and $10 \mathrm{wt} \% \mathrm{Ni}$ composite KS10 was previously observed by Funabiki et al. ${ }^{7,26}$ This sharp change in the diffusion coefficient occurs in the region of the coexistence of stage 4 and dilute stage 1 biphase. ${ }^{7}$ The lithium kinetics at the biphasic state are believed to be governed by the displacement rate of the biphasic interface and not by the Fickian diffusion process.

Self-discharge processes normally take place during a highly charged state of the electrode and are caused by the highly oxidizing and reducing character of the electrode materials and the electrolyte, respectively. ${ }^{18}$ Self-discharge is also mainly associated with the performance of the negative electrode, although it can be associated with the positive electrode, but to a much lesser extent. ${ }^{23}$ Therefore, self-discharge of a Li-ion cell is largely attributed to the loss of capacity in the graphite electrode, in which reversible delithiation takes place in conjugation with irreversible solvent reduction. ${ }^{23,24}$ In other words, the self-discharge process can be indicative of the stability of the electrolyte solution toward the graphite electrode. It is generally observed that Li-ion cells comprised of a negative electrode/electrolyte system that produces a poor solid electrolyte interphase (SEI) film on the electrode surface, often gives rise to the highest rate of self-discharge or largest capacity loss. ${ }^{23}$

To investigate the stability of the SEI film formed on the surfaces of both bare KS10 and 10 wt \% Ni composite KS10, the self-discharge behavior of these materials was tested by determining the discharge capacity retained after different storage times. Figure 7 shows the percentage of the discharge capacity retained for both materials after storing them for 1,3 , and 10 days. After 1 day of storage, 2 to $3 \%$ of the capacity was lost, after 3 days of storage 5 to $6 \%$ of the capacity was lost, and after 10 days of storage 8 to $10 \%$ of the capacity was lost. However, in all cases, the higher lost capacity percentages are associated with the bare KS10 graphite. In other words, the capacity losses due to self-discharge are reduced by coating KS10

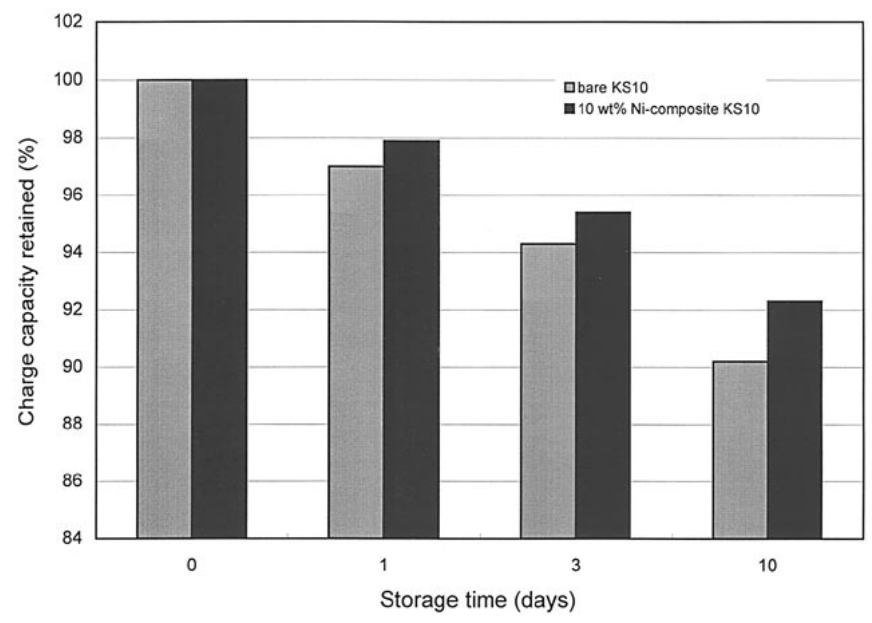

Figure 7. Self-discharge behavior of bare KS10 and 10 wt \% Ni composite KS10. 
graphite with 10 wt $\% \mathrm{Ni}$ composite. This slightly lower self-discharge rate associated with the $10 \mathrm{wt} \% \mathrm{Ni}$ composite KS10 may be attributed to the formation of a more stable, but not more resistant, SEI film (see Fig. 5).

\section{Conclusions}

Part I of this series reported on the development of a novel electroless deposition technique for making $\mathrm{Ni}$ composite KS10 graphite. When investigated as the negative electrode in a Li-ion cell with PC based electrolyte, a 10 wt \% Ni composite KS10 graphite exhibited a marked improvement in the charge-discharge performance, coulombic efficiency and cycling behavior compared to the bare precursor graphites. In the present study, i.e., Part II of this series, electrochemical impedance and self-discharge studies were carried out to investigate kinetic effects associated with coating KS10 graphite with 10 wt \% Ni composite. Charge-transfer resistances, exchange current densities, surface film resistances, and lithium-ion diffusion coefficients as functions of the SOC were extracted from impedance spectra obtained for bare KS10 and $10 \mathrm{wt} \%$ Ni composite KS10. The charge-transfer resistances obtained for the $10 \mathrm{wt} \% \mathrm{Ni}$ composite KS10 were always lower than those obtained for the bare KS10, and gave rise to between 26 and 27\% larger exchange current densities for the $10 \mathrm{wt} \% \mathrm{Ni}$ composite KS10, which increased from 137 to $614 \mathrm{~mA} / \mathrm{g}$ as the SOC increased. This result indicated that a $10 \mathrm{wt} \% \mathrm{Ni}$ composite coating is favorable for the lithium-ion charge-transfer reaction. The surface film resistances for Ni composite KS10 were between 0.02 and $0.05 \Omega \mathrm{g}$, slightly smaller than those of 0.03 to $0.08 \Omega \mathrm{g}$ for bare KS10, and both surface film resistances decreased with increasing SOC. This result suggested that that the $10 \mathrm{wt} \% \mathrm{Ni}$ composite coating on KS10 graphite possibly enhanced the lithium-ion conduction in the surface film. The lithium-ion diffusion coefficients extracted from the transition diffusion region in the Nyquist plots were always slightly larger for the $10 \mathrm{wt} \% \mathrm{Ni}$ composite KS10 compared to the bare KS10, ranging between $1.1 \times 10^{-9}$ and $6.7 \times 10^{-9} \mathrm{~cm}^{2} / \mathrm{s}$, and $1.28 \times 10^{-9}$ and $7.1 \times 10^{-9} \mathrm{~cm}^{2} / \mathrm{s}$, respectively. These results show very clearly that the $10 \mathrm{wt} \% \mathrm{Ni}$ composite coating on KS10 graphite did not hinder the diffusion of lithium ions into the graphite structure, and in most cases it had a beneficial effect. Both materials also exhibited an increase in the lithium-ion diffusion coefficient at higher SOCs; this behavior was explained in terms of the formation of a graphite-intercalation compound Li-GIC in-plane superstructure, lower local strains between the graphene layers, and larger expansion of the graphene layers, as more lithium-ions intercalated into the graphite. Results from a self-discharge study also favored the $10 \mathrm{wt} \% \mathrm{Ni}$ composite KS10, which exhibited less capacity loss over a 10 day period compared to bare KS10. This result was attributed to the formation of a more stable, but not more resistant, SEI film on the surface of the KS10 coated with 10 wt \% Ni composite.

\section{Acknowledgments}

Financial support provided in part by the DOE Division of Chemical Sciences, Office of Basic Energy Sciences, G. M. DeFG02-96ER 146598, in part by the Office of Research and Development, C/O no. 93-F148100-100 and in part by the Army Research Office, grant no. DAAH04-96-1-0421 are gratefully acknowledged.
The University of South Carolina assisted in meeting the publication costs of this article.

\section{List of Symbols}

$C_{\mathrm{dl}} \quad$ double-layer capacitance, $\mu \mathrm{F}$

$C_{\mathrm{sl}} \quad$ surface film capacitance, $\mu \mathrm{F}$

D lithium ion diffusion coefficient in graphite, $\mathrm{cm}^{2} / \mathrm{s}$

F Faraday's constant, 96,487 Clequiv

I current, A

$j \quad$ imaginary number, $\sqrt{-1}$

$R \quad$ gas constant, $8.314 \mathrm{~J} / \mathrm{mol} \mathrm{K}$

$R_{\mathrm{p}} \quad$ radius of spherical particle, $\mathrm{cm}$

$R_{\mathrm{ct}} \quad$ charge-transfer resistance, $\Omega \mathrm{g}$

$R_{\mathrm{S}} \quad$ electrolyte ionic resistance, $\Omega \mathrm{g}$

$R_{\mathrm{sl}} \quad$ surface film resistance, $\Omega \mathrm{g}$

$x \quad \mathrm{Li}_{x} \mathrm{C}_{6}$ stoichiometric parameter, mole

$Z_{\mathrm{Im}} \quad$ imaginary impedance, $\Omega \mathrm{g}$

$Z_{\mathrm{Re}} \quad$ real impedance, $\Omega \mathrm{g}$

$Z_{\mathrm{w}} \quad$ Warburg impedance, $\Omega \mathrm{g}$

$\omega \quad$ angular frequency s ${ }^{-1}$

$\sigma \quad$ modified Warburg prefactor, $\Omega / \mathrm{s}^{1 / 2}$

$\eta_{\mathrm{R}} \quad$ particle surface overpotential $(r=R), \mathrm{V}$

$\psi \quad$ defined by Eq. 8

$\partial \eta_{\mathrm{R}} / \partial I \quad$ nondiffusion impedance, $\Omega$

\section{References}

1. H. Shi, J. Barker, M. Y. Saidi, and R. Koksbang, J. Electrochem. Soc., 143, 3466 (1996).

2. J. R. Dahn, R. Fong, and M. J. Spoon, Phys. Rev. B, 42, 6424 (1990).

3. R. Yazami and M. Deschamps, Bull. Electrochem., 12, 206 (1996).

4. T. D. Tran, J. H. Feikert, X. Song, and K. Kinoshita, J. Electrochem. Soc., 142, 3297 (1995).

5. S. R. Narayanan, D. H. Shen, and G. Halpert, J. Electrochem. Soc., 140, 1854 (1993).

6. J. Xu and G. C. Farrington, J. Electrochem. Soc., 142, 3303 (1995)

7. A. Funabiki, M. Inaba, and Z. Ogumi, J. Electrochem. Soc., 145, 172 (1998).

8. K. Kinoshita, Carbon Electrochemical and Physicochemical Properties, John Wiley \& Sons Inc., New York (1988).

9. P. Yu, J. A. Ritter, R. E. White, and B. N. Popov, J. Electrochem. Soc., 147, 1280 (2000).

10. B. Markovsky, M. D. Levi, and D. Aurbach, Electrochim. Acta, 43, 2287 (1998).

11. P. Yu, B. N. Popov, J. A. Ritter, and R. E. White, J. Electrochem. Soc., 146, 8 (1999).

12. B. Haran, B. N. Popov, and R. E. White, J. Power Sources, 75, 56, (1998)

13. K. Naoi, M. Mori, Y. Naruoka, W. M. Lamanna, and R. Atanasoski, J. Electrochem. Soc., 146, 462 (1999).

14. Handbook of Chemistry and Physics, 52nd ed., R. C. Weast, Editor, The Chemical Rubber Co., Cleveland, OH (1971).

15. A. J. Bard and L. R. Faulkner, Electrochemical Methods-Fundamentals and Applications, John Wiley \& Sons Inc., New York (1980).

16. J. S. Newman, Electrochemical Systems, 2nd ed., Prentice Hall International., Englewood Cliffs, NJ (1991)

17. H. Kanoh, Q. Feng, Y. Miyai, and K. Ooi, J. Electrochem. Soc., 142, 702 (1995)

18. D. Guyomard and J. M. Tarascon, J. Electrochem. Soc., 139, 937 (1992).

19. N. Takami, A. Satoh, M. Hara, and T. Ohsaki, J. Electrochem. Soc., 142, 371 (1995).

20. T. Uchida, Y. Morikawa, H. Ikuta, and M. Wakihara, J. Electrochem. Soc., 143, 2606 (1996).

21. C. Julien and G. Nazri, Solid State Batteries: Materials Design and Optimization, p. 392, Kluwer Academic Publishers, Boston, MA (1994).

22. M. S. Dresselhaus and G. Dresselhaus, Adv. Phys., 30, 139 (1981).

23. M. C. Smart, B. V. Ratnakumar, and S. Surampudi, J. Electrochem. Soc., 146, 486 (1999).

24. P. Arora, B. N. Popov, and R. E. White, J. Electrochem. Soc., 145, 807 (1998).

25. S. Shiraishi, K. Kanamura, and Z. Takehara, J. Electrochem. Soc., 146, 1633 (1999).

26. N. Takami, A. Satoh, T. Ohsaki, and M. Kanda, J. Electrochem. Soc., 145, 478 (1998) 